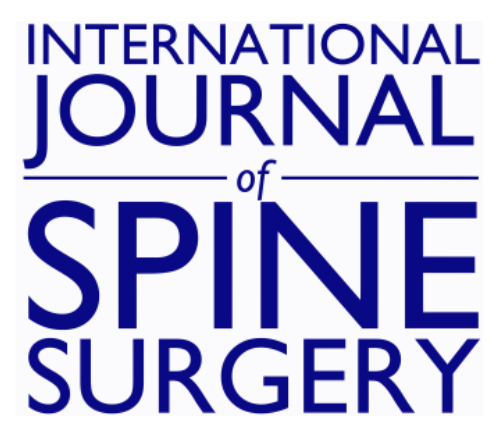

\title{
Use of O-arm With Intraoperative Arteriography for Localization and Stealth Navigation of the Vertebral Arteries During Posterior Cervical Spine Surgery
}

J. Willis Kiessling, Ajay Ramnot, Tiffany Odell, Yasir Khan and Deependra Mahato

Int J Spine Surg 2021, 14 (s4) S10-S15

doi: https://doi.org/10.14444/7158

http://ijssurgery.com/content/14/s4/S10

This information is current as of April 26, 2023.

Email Alerts Receive free email-alerts when new articles cite this article. Sign up at:

http://ijssurgery.com/alerts

The International Journal of Spine Surgery

2397 Waterbury Circle, Suite 1,

Aurora, IL 60504, Phone: +1-630-375-1432 


\title{
Use of O-arm With Intraoperative Arteriography for Localization and Stealth Navigation of the Vertebral Arteries During Posterior Cervical Spine Surgery
}

\author{
J. WILLIS KIESSLING, DO, AJAY RAMNOT, DO, TIFFANY ODELL, DO, YASIR KHAN, MBBS, \\ DEEPENDRA MAHATO, DO, MS \\ Department of Neurosurgery, Desert Regional Medical Center, Palm Springs, California
}

\begin{abstract}
Background: Vertebral artery injury (VAI) can be a devastating complication during cervical spine surgery. Although considered a rare occurrence overall, incidences of VAI have been reported in the ranges of $0.07 \%$ to $8 \%$. Such injuries have the potential for catastrophic consequences, including blood loss, permanent morbid neurologic injury, and even death. The introduction of intraoperative navigation using either preoperative or intraoperative imaging has now been widely adopted in current practice so as to try and minimize adverse outcomes while giving realtime, dynamic information of the operative field. The use of the O-arm Surgical Imaging System during cervical spine surgery allows one to obtain high-resolution, accurate intraoperative imaging, and when used in concert with forms of intraoperative navigation, it can help with instrumentation and safety. However, patients undergoing cervical spine surgery do not routinely undergo preoperative vascular imaging, particularly with regard to anterior cervical or posterior high-cervical surgeries, where the incidence of VAI, in comparison with other cervical surgeries, has been reported to be the highest.

Methods: Here we present the use of intraoperative O-arm-based arteriography for integration with navigation for vertebral artery localization during $\mathrm{C} 1$ to $\mathrm{C} 3$ posterior instrumentation and fusion of an unstable $\mathrm{C} 2$ fracture in a 54year-old man.

Results: The patient did not experience any intraoperative VAI and was subsequently discharged with no focal neurologic deficits.

Conclusions: Detailed in our report is our protocol and procedure for obtaining and using intraoperative angiographic images.

Clinical Relevance: Case report detailing O arm for intraoperative identification of vertebral arteries during C1C3 posterior instrumentation and fusion with pre-operative unilateral vertebral artery injury.

Cervical Spine

Keywords: O-arm, stealth navigation, vertebral artery injury
\end{abstract}

\section{INTRODUCTION}

Vertebral artery injury (VAI) is a known complication that can occur during cervical spine surgery. VAI has been reported to occur in the range of $0.07 \%$ to $8 \% .^{1-3}$ Reports of neurologic deficit and death are reported as low as $0.14 \%$, although the ranges have been estimated to be between 0 and $33 \% .^{1,4-6}$ The most commonly reported cause is that of abnormal vertebral artery anatomy, and the importance of preoperative planning and imaging of the vertebral arteries has been extolled in nearly every publication on VAI after cervical spine surgery. ${ }^{1-6}$ The use of intraoperative navigation on a preoperative or intraoperative basis has become routine in practices that have the technology available. This is true in spine surgery, especially with regard to high cervical instrumentation. The use of the O-arm Surgical Imaging System (Medtronic, Dublin, Ireland) has routinely been used to facilitate a safer dissection and for guidance of instrumentation. Although the safety of vascular structures during high cervical fusions is paramount, vessel imaging preoperatively is not routinely available. ${ }^{1-4}$ Detailed below is a case report of the use of intraoperative O-arm-based arteriography for integration with navigation for vertebral artery localization during $\mathrm{C} 1$ to $\mathrm{C} 3$ posterior instrumentation and fusion of an unstable $\mathrm{C} 2$ fracture in a 54year-old man. 

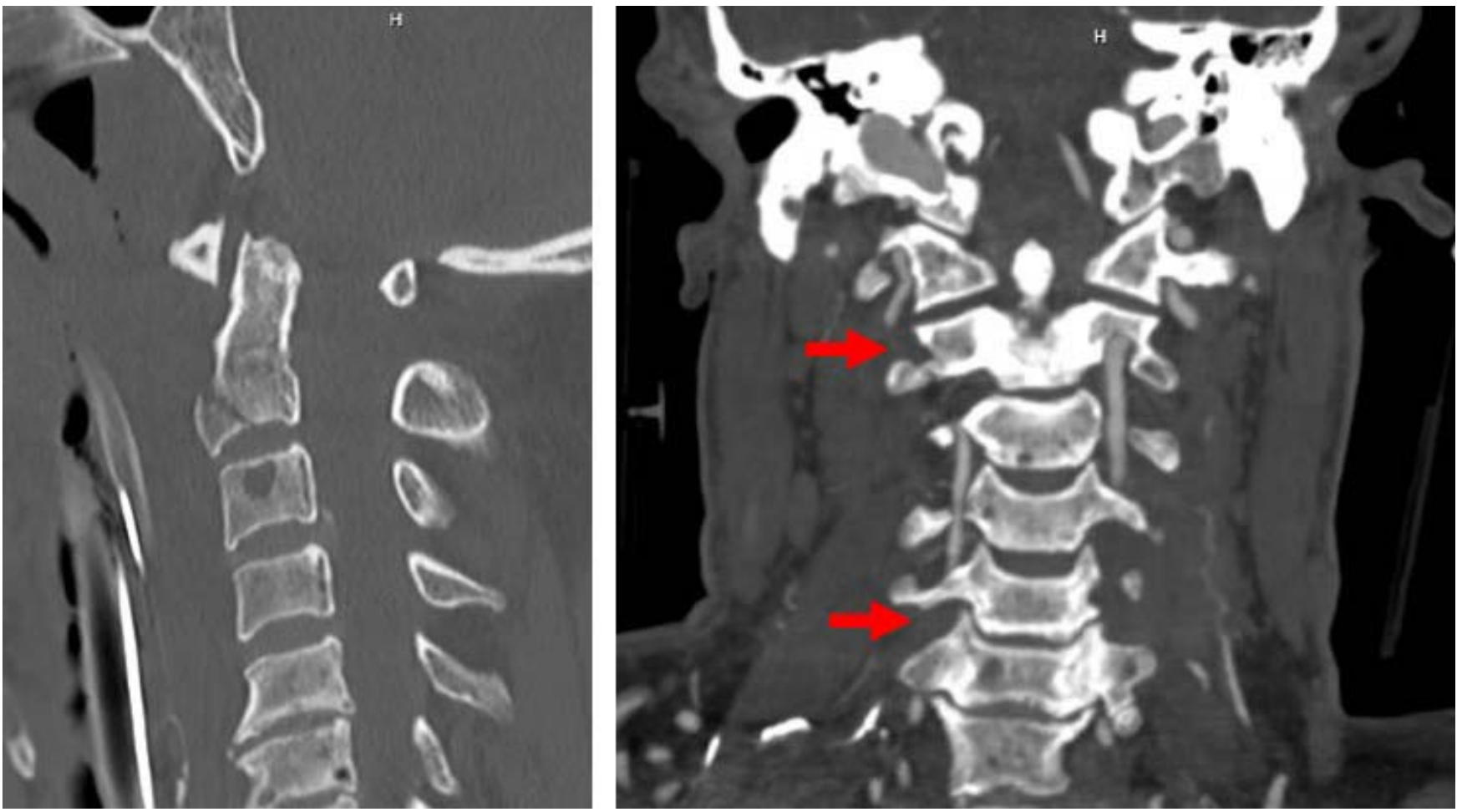

Figure 1. (Left) Sagittal computed tomography (CT) cervical spine demonstrating anterior inferior C2 vertebral body fracture. (Right) Coronal CT angiography of the neck with arrows demonstrating levels of right vertebral artery injury at C2 and C6.

\section{CASE REPORT}

A 54-year-old male bicyclist with unknown past medical history was struck at approximately $50 \mathrm{mph}$ by a vehicle. On exam the patient was awake, albeit agitated, and moving all 4 extremities with no neurologic deficit. Workup revealed a type $3 \mathrm{C} 2$ fracture with mild retrolisthesis of $\mathrm{C} 2$ on $\mathrm{C} 3$ and extension into the right foramen transversarium as well as destabilizing posterior ligamentous and bilateral $\mathrm{C} 1$ to $\mathrm{C} 2$ facet injury (Figure 1). Computed tomography angiography (CTA) showed a dominant left vertebral artery that was uninjured. However, the right vertebral artery was found to have occlusion with distal reconstitution noted at C6 and again at the level of injury at C2 (Figure 2). Given the extensive ligamentous injury, the patient was taken to the operating room for planned $\mathrm{C} 1$ to $\mathrm{C} 3$ posterior instrumentation and fusion. Preoperative planning included the use of O-arm with intraoperative contrast administration to integrate it into the Medtronic Stealth Navigation System (Dublin, Ireland) for intraoperative navigation and localization so as to maximize safety during the surgery for the patient's dominant left vertebral artery. The patient's exam continued to be without neurologic deficit, and the patient had no evidence of stroke.

The use of Isovue (Bracco, Milan) as a contrast medium and dosing for CTA neck has been well documented. ${ }^{7,8}$ Prior to surgery, the rate of Isovue injection as well as safety parameters were discussed with radiologists and radiology technologists to optimize the radiographic results while maintaining the highest level of patient safety. The patient was placed in a Mayfield pin head-holder; once he was positioned prone, the O-arm was brought in for localization over the occipital-atlanto-axial spine and planned arteriography. The Medtronic ${ }^{\mathrm{TM}}$ Stealth Navigation System was attached to the Mayfield as well.

After confirmation of appropriate positioning with intraoperative fluoroscopy, the patient then received $100 \mathrm{~mL}$ of Isovue 370 contrast intravenously, via an autoinfuser, at the rate of $4 \mathrm{~mL} / \mathrm{s}$. The injection was done through a right antecubital 16gauge IV. On initiation of the O-arm for CT imaging, the time from start to actual imaging processing was 8 seconds. This was an important nuance to consider because the ideal time from contrast injection to imaging was 25 seconds. Thus, the start of the O-arm imaging needed to take this 


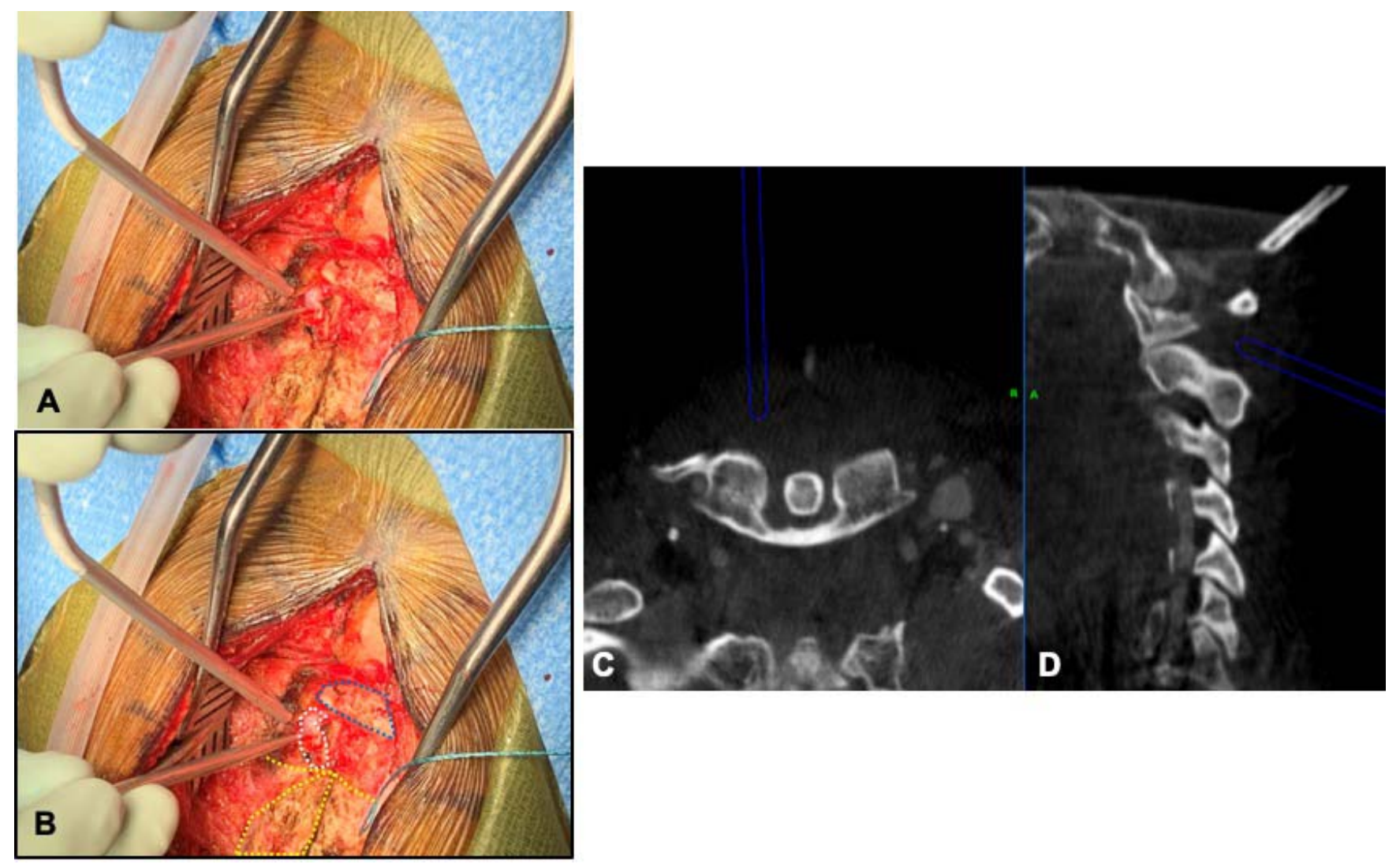

Figure 2. Correlation of finding of vascular structure (A and B) intraoperatively. O-arm imaging showing both axial (C) and sagittal (D) navigation. Dashed blue line is C1 posterior arch, dashed yellow line is C2 body, and dashed white line is vascular structure.

into account and was initiated 17 seconds after injection.

Once completed, 3D reconstruction of the bilateral vertebral arteries was able to be constructed and viewed with the $\mathrm{O}$-arm, and the arteriography was integrated into the CT images for real-time localization with Medtronic Stealth Navigation System intraoperatively. Thus, prior to incision, the course of the vertebral arteries could be easily assessed for any abnormal course prior to incision. In addition, real-time localization of the vertebral arteries could be obtained at any time and through the entire surgery. This was used to help guide microdropper assessment and interpretation during the surgery as well (Figures 3 and 4).

Bilateral lateral mass screws were placed in $\mathrm{C} 1$ and $\mathrm{C} 3$ and pedicle screws were placed in $\mathrm{C} 2$, all under navigation and with the vertebral arteries localized at all times. There were no complications during the surgery, and the patient awoke with no deficits and continued intact neurologic exam and was returned to the intensive care unit for continued trauma management.

\section{DISCUSSION}

VAI during cervical spine surgery continues to have the potential for devastating complications. Although incidences are relatively low, the most common cause has been reported as due to abnormal vertebral artery anatomy that is not appreciated or found during preoperative workup and planning. ${ }^{1-4}$ Lunardini et al, ${ }^{1}$ in a presentation of their findings on questionnaires completed by members of the international Cervical Spine Research Society, reported the highest occurrences of VAI in cervical spine surgery coming with posterior instrumentation of the upper cervical spine, anterior corpectomy, and posterior exposure of the cervical spine. Although several associations were reported with regard to causes of VAI during cervical spine surgery, they reported that 22 of 111 VAIs involved an anomalous course of the vertebral artery. ${ }^{1}$ This may represent a variable in cervical spine surgery that, with proper imaging and evaluation, could substantially reduce the risk for VAI.

In our patient, his workup revealed injury to the bony structures known to contain the vertebral arteries, and thus he underwent CTA for evaluation 


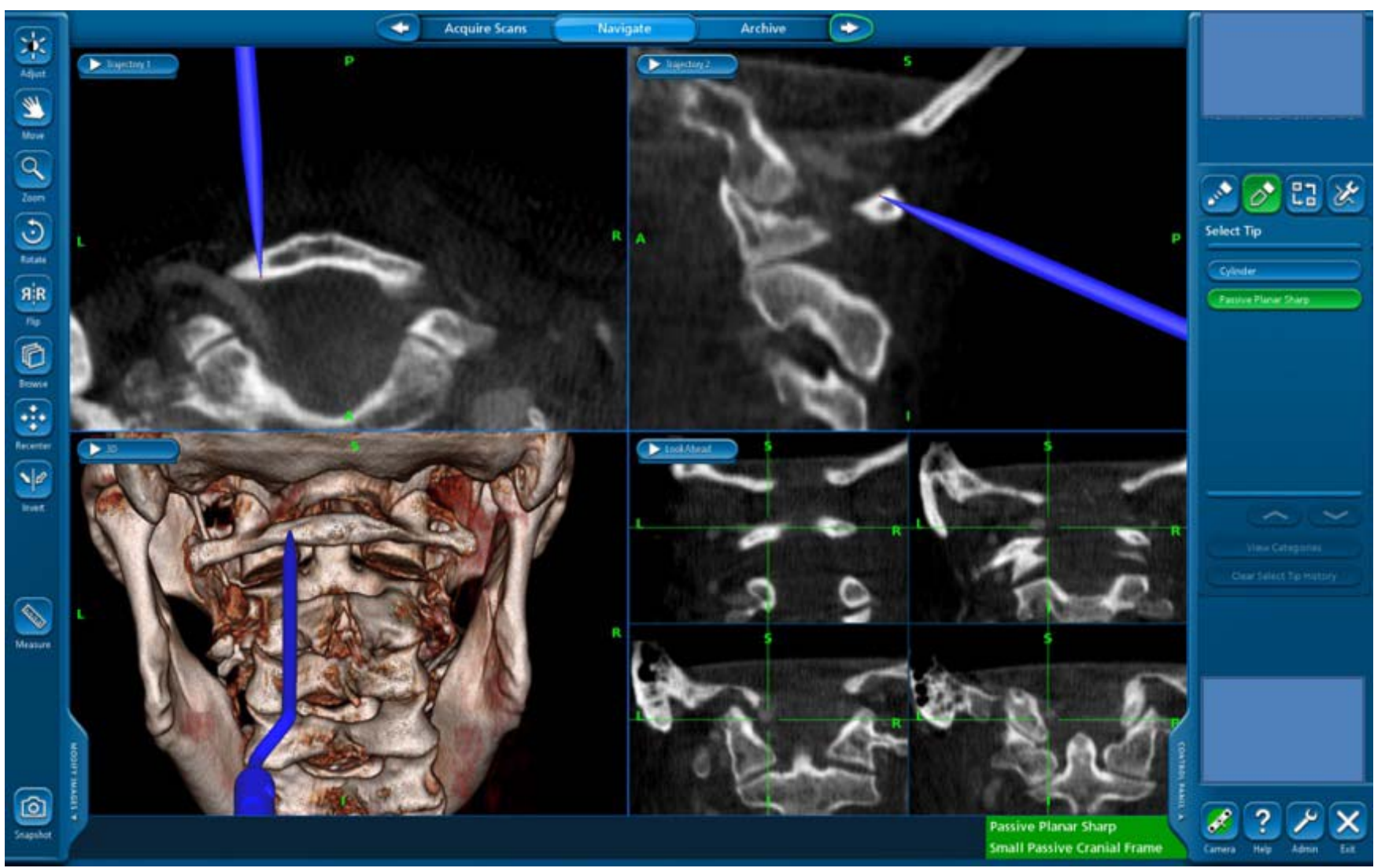

Figure 3. Examples of intraoperative navigation after intraoperative arteriography to find location of the vertebral artery.

of arterial injury. However, most patients undergoing cervical spine surgery, whether because of trauma or degenerative processes, do not undergo preoperative vascular imaging unless there exists pathology or history to indicate doing so. The use of intraoperative $\mathrm{O}$-arm with contrast administration serves to not only provide critical information on the anatomy and location of the cervical vasculature, it also integrates the angiography findings into the high-resolution CT images, which allows for highly accurate localization with navigation. In our case, Medtronic Stealth Navigation System was used, and once the cranial navigation frame was placed on the Mayfield head-holder, the O-armbased images and arteriography were obtained before the patient was even prepped and draped, allowing for immediate review and visualization of the vertebral arteries. With its integration into the Medtronic Stealth Navigation System, vertebral vasculature could be localized at all times during the dissection and during screw placement, thereby potentially mitigating 2 of the most commonly reported causes of VAI. In addition, no additional radiation exposure was necessary because the patient was already to receive $\mathrm{O}$-arm imaging, with the biggest increased risk coming from the contrast injection. It has been reported that the incidence of contrast-induced kidney injury in elective patients can be less than $1 \%$, and even with its risk increased in the setting of critically ill patients, with smaller contrast loads of 30 to $125 \mathrm{~mL}$ this can be further reduced. ${ }^{9}$ Elderly populations defined as older than 65 years also have an increased risk of contrastinduced AKI. ${ }^{10}$ Renal dosing or avoidance of contrast depending on the severity of renal impairment will have to be implemented. Although we believe this technique substantially improves the risk profile in cervical spine surgery, we do recognize that perhaps many patients do not necessarily need $\mathrm{O}$-arm-based navigation or imaging. We do not advocate the use of O-arm strictly for any patients undergoing cervical spine surgery just for the sake of vascular imaging. However, if $\mathrm{O}$-arm use has been planned for use anyway, this serves as a minimal workflow addition with minimal impacts on overall operative time and with substantial benefits to planning and real-time intraoperative anatomic knowledge. We feel this technique is even more beneficial in the setting of high-risk surgeries in high-risk patients. As reported previously, the most 


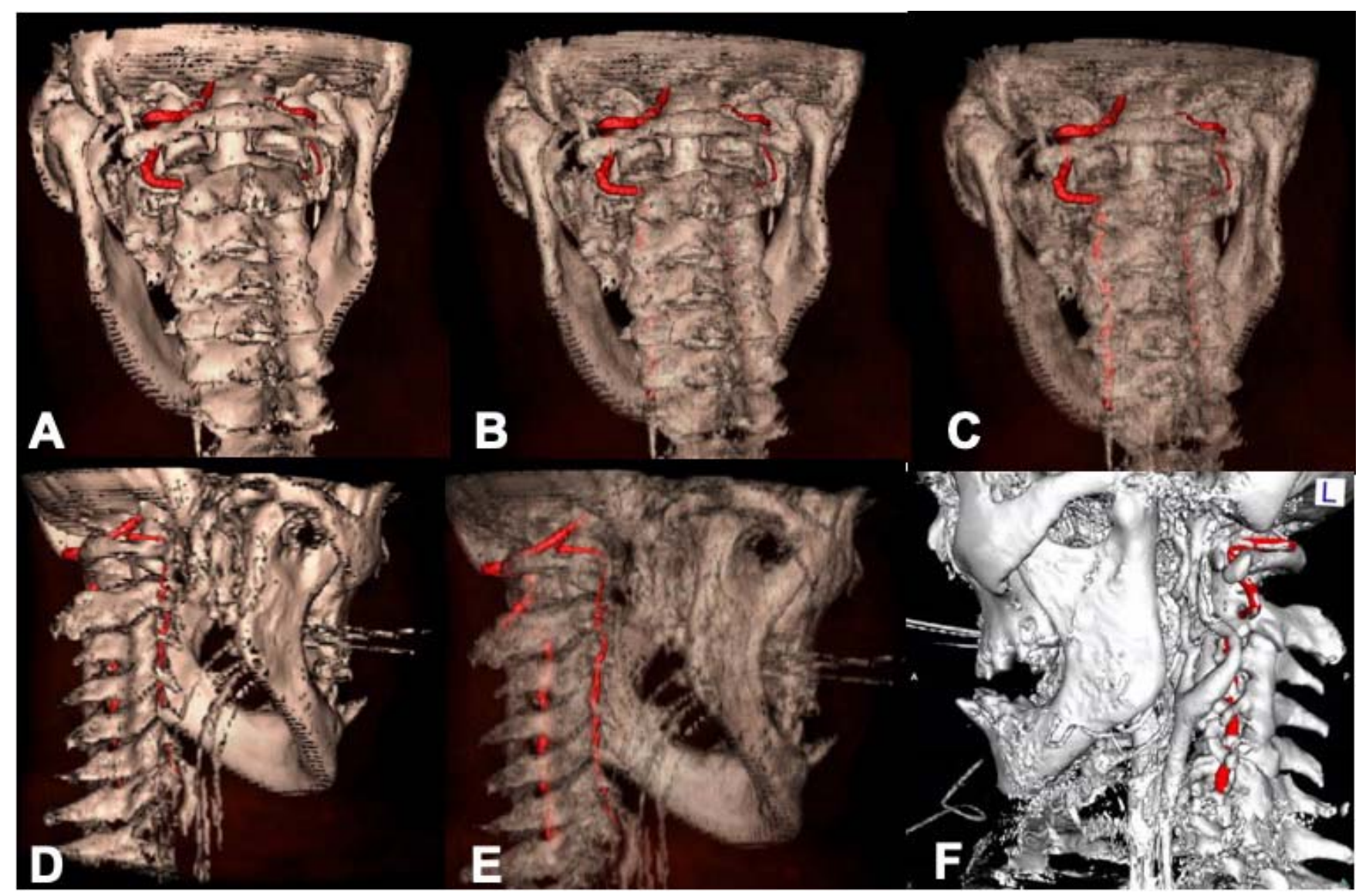

Figure 4. Three-dimensional (3D) reconstructions performed with O-arm intraoperatively displaying vertebral artery course. (A) Original 3D reconstruction on O-arm. ( $B$ and $C)$ Progressive intraoperative image alterations made so as to see the entire course of the vertebral arteries with the cervical spine. (D) In comparison with the intact, dominant, left vertebral artery, the injured right vertebral artery and its course are imaged. (E) Once again, the entire course of the right vertebral artery can be merged and reconstructed from the O-arm arteriography. (F) High-resolution reconstruction showing the course of the left vertebral artery in the upper cervical spine.

at-risk surgeries for VAI are upper cervical instrumentation ( $\mathrm{C} 1$ to $\mathrm{C} 3$ region) and anterior corpectomies, followed somewhat distantly by posterior cervical dissection. ${ }^{1,3,4}$ Our patient was high risk because he had already experienced 2 right VAIs from his trauma. Fortunately, he was left vertebral artery dominant, but this further necessitated accurate, methodical preoperative planning so as to minimize the VAI risk as much as possible. With this technique, the left vertebral artery could be found at any time during the surgery and, most critically, with lateral mass and pedicle screw placement.

Another consideration for the use of this technique is with regard to teaching institutions. Ours is a teaching institution where neurosurgical residents are critical and integral in the care and operative planning of patients as well as with their surgeries. Although there is no published literature to date regarding the incidence of VAI due to residents versus attendings, Lunardini et $\mathrm{al}^{1}$ did report an association between higher incidences of VAI in those surgeons who had performed fewer than 300 cervical spine surgeries versus those who had performed 300 or more $(0.33 \%$ versus $0.06 \%$, respectively). Although residents are operating under the direct supervision of an experienced attending, the risks of VAI while a resident performs the critical portions of the case are likely, and expectedly, higher. This modality, which enhances real-time vascular localization and anatomic navigation, provides substantial feedback to the resident and improves their confidence regarding the operative anatomy and technique while potentially reducing substantially the risk of VAI and its related complications. In dialogue and informed consent with patients, it may even enhance the patient's experience and their perception of safety, knowing that these critical structures can be localized and protected at all times during the case.

VAI during cervical spine surgery is a known and potentially avoidable complication. Although many 
different methods of imaging, navigation, or assessment can be used to minimize this risk, it will never be a substitute for anatomic knowledge and preparation of the individual surgeon. Fluoroscopy, $\mathrm{O}$-arm, navigation, neuromonitoring, and preoperative imaging are all adjuncts to help support the base knowledge the practitioner should already have in the performance of these surgeries, but for many they have helped with the performance of increasingly safer surgeries. We feel the use of intraoperative angiography in conjunction with the $\mathrm{O}$-arm adds a small additional step with minimal adverse risk to the patient undergoing cervical spine surgery while optimizing and enhancing patient safety.

\section{ACKNOWLEDGMENTS}

The authors give special thanks to Dan Blais for his assistance in the planning and performance of this procedure, as well as his invaluable experience with navigation, $\mathrm{O}$-arm, and computer modeling.

\section{REFERENCES}

1. Lunardini DJ, Eskander MS, Even JL, et al. Vertebral artery injuries in cervical spine surgery. Spine $J$. 2014;14(8):1520-1525.

2. Schroeder GD, Hsu WK. Vertebral artery injuries in cervical spine surgery. Surg Neurol Int. 2013;4(suppl 5):S362S367.

3. Molinari RW, Chimenti PC, Molinari R Jr, Gruhn W. Vertebral artery injury during routine posterior cervical exposure: case reports and review of literature. Global Spine J. 2015;5(6):528-532.

4. Molinari R, Bessette M, Raich AL, Dettori JR, Molinari C. Vertebral artery anomaly and injury in spinal surgery. Evid Based Spine Care J. 2014;5(1):16-27.

5. Kiessling JW, Whitney E, Fiani B, Khan YR, Mahato D.
C2-3 fracture dislocation and bilateral vertebral artery occlusion without neurological injury: a case report. Cureus. 2019;11(8):e5538. doi:10.7759/cureus.5538

6. Eskander MS, Drew JM, Aubin ME, et al. Vertebral artery anatomy: a review of two hundred fifty magnetic resonance imaging scans. Spine (Phila Pa 1976). 2010;35(23):2035-2040.

7. Zhang WL, Li M, Zhang B, et al. CT angiography of the head-and-neck vessels acquired with low tube voltage, low iodine, and iterative image reconstruction: clinical evaluation of radiation dose and image quality. PLoS One. 2013;8(12):e81486. doi:10.1371/journal.pone.0081486

8. Zhang B, Dong $\mathrm{Y}$, Liang $\mathrm{L}$, et al. The incidence, classification, and management of acute adverse reactions to the low-osmolar iodinated contrast media Isovue and Ultravist in contrast-enhanced computed tomography scanning. Medicine (Baltimore). 2016;95(12):e3170. doi:10.1097 MD.0000000000003170

9. Ozkok S, Ozkok A. Contrast-induced acute kidney injury: a review of practical points. World J Nephrol. 2017;6(3):86-99.

10. Song W, Zhang T, Pu J, Shen L, He B. Incidence and risk of developing contrast-induced acute kidney injury following intravascular contrast administration in elderly patients. Clin Interv Aging. 2014;9:85-93.

Disclosures and COI: The authors report no conflict of interest concerning the materials or methods used in this case or review for this paper.

Corresponding Author: Ajay Ramnot, DO, 1080 North Indian Canyon Drive, Suite 201, Palm Springs, CA 92262. Phone: (954) 383-7650; Email: Ajramnot@gmail.com.

Published 11 February 2021

This manuscript is generously published free of charge by ISASS, the International Society for the Advancement of Spine Surgery. Copyright @ 12020 ISASS. To see more or order reprints or permissions, see http://ijssurgery.com. 